\title{
Metabolic activity of Streptococcus mutans biofilms and gene expression during exposure to xylitol and sucrose
}

\author{
Eva-Maria Decker ${ }^{1}$, Christian Klein ${ }^{1,2}$, Dimitri Schwindt ${ }^{1}$ and Christiane von Ohle ${ }^{1}$
}

The objective of the study was to analyse Streptococcus mutans biofilms grown under different dietary conditions by using multifaceted methodological approaches to gain deeper insight into the cariogenic impact of carbohydrates. $S$. mutans biofilms were generated during a period of $24 \mathrm{~h}$ in the following media: Schaedler broth as a control medium containing endogenous glucose, Schaedler broth with an additional $5 \%$ sucrose, and Schaedler broth supplemented with $1 \%$ xylitol. The confocal laser scanning microscopy (CLSM)-based analyses of the microbial vitality, respiratory activity (5-cyano-2,3-ditolyl tetrazolium chloride, CTC) and production of extracellular polysaccharides (EPS) were performed separately in the inner, middle and outer biofilm layers. In addition to the microbiological sample testing, the glucose/sucrose consumption of the biofilm bacteria was quantified, and the expression of glucosyltransferases and other biofilm-associated genes was investigated. Xylitol exposure did not inhibit the viability of $S$. mutans biofilms, as monitored by the following experimental parameters: culture growth, vitality, CTC activity and EPS production. However, xylitol exposure caused a difference in gene expression compared to the control. GtfC was upregulated only in the presence of xylitol. Under xylitol exposure, gtfB was upregulated by a factor of 6 , while under sucrose exposure, it was upregulated by a factor of three. Compared with glucose and xylitol, sucrose increased cell vitality in all biofilm layers. In all nutrient media, the intrinsic glucose was almost completely consumed by the cells of the $S$. mutans biofilm within $24 \mathrm{~h}$. After $24 \mathrm{~h}$ of biofilm formation, the multiparametric measurements showed that xylitol in the presence of glucose caused predominantly genotypic differences but did not induce metabolic differences compared to the control. Thus, the availability of dietary carbohydrates in either a pure or combined form seems to affect the cariogenic potential of $S$. mutans biofilms.

International Journal of Oral Science (2014) 6, 195-204; doi:10.1038/ijos.2014.38; published 25 July 2014

Keywords: biofilms; 5-cyano-2,3-ditolyl tetrazolium chloride; extracellular polysaccharides; gene expression; Streptococcus mutans; sucrose; viability; xylitol

\section{INTRODUCTION}

Similar to other bacteria, oral microorganisms have a strong tendency to attach to liquid/solid interfaces and colonize them. Furthermore, as a survival mechanism, they grow as complex communities called biofilms. The genotypic and phenotypic expression profiles of biofilm bacteria differ strongly from those of their planktonic counterparts. For example, biofilm bacteria have increased antimicrobial resistance, are regulated by quorum sensing, and form a distinctive extracellular polymeric matrix network. ${ }^{1-3}$ Dental biofilm formation can lead to the development of oral infectious diseases, such as caries, gingivitis and periodontal inflammation. Streptococcus mutans belongs to a group of colonizers of human teeth and has the ability to metabolize various carbohydrates into organic acids, which may lead to the cariogenic destruction of tooth surfaces. ${ }^{4}$ In particular, sucrose, the substrate for glucosyltransferase-mediated sucrose-dependent glucan production, promotes the adhesion of $S$. mutans to tooth surfaces. ${ }^{5-6}$ Sucrose is a fermentable disaccharide and may act as a substrate for extracellular polysaccharide synthesis. Changes in the local environment at the tooth surface induced by sucrose exposure disturb the microbial balance and may promote the growth of acidogenic populations including S. mutans, actinomyces and lactobacilli. ${ }^{7}$ Substituting nutritive sweeteners for cariogenic sugars is an important measure for caries prevention in oral hygiene care, similar to chemotherapeutic treatment and fluoridation. Polyol xylitol cannot be metabolized into acids by oral microorganisms for energy generation; instead, after cellular uptake, it accumulates in the cell as a toxic sugar-phosphate. ${ }^{8}$ The architecture and gene expression profiles of $S$. mutans biofilms are altered according to the availability of sugar. ${ }^{9-10}$

The cariogenic properties of $S$. mutans biofilms are regulated by various genes, which are involved in five essential metabolic pathways: (i) microbial adhesion, ${ }^{11-13}$ (ii) biofilm formation, ${ }^{14-16}$ (iii) extracellular polysaccharide synthesis, ${ }^{12-13}$ (iv) carbohydrate uptake, ${ }^{17-18}$ and (v) acid tolerance. ${ }^{19}$

Several clinical studies have focused on the effects of xylitol on $S$. mutans, but the results are contradictory. While various investigations showed the plaque-reducing effects of xylitol, ${ }^{20-22}$ other studies could not confirm these results. ${ }^{23-24}$ Generally, clinical investigations of biofilms are complicated by the heterogeneity of the oral environment,

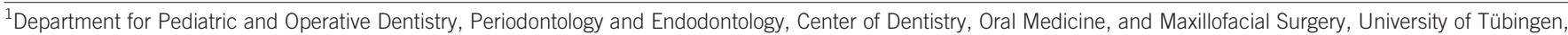
Tübingen, Germany and ${ }^{2}$ Private Practice Zahngesundheit Waiblingen, Waiblingen, Germany

Correspondence: Dr EM Decker, Department for Pediatric and Operative Dentistry, Periodontology and Endodontology, Center of Dentistry, Oral Medicine, and Maxillofacial

Surgery, University of Tübingen, Osianderstrasse 2-8, Tübingen D-72076, Germany

E-mail: evi.decker@med.uni-tuebingen.de

Accepted 3 June 2014 
the distribution of carbohydrates, and the complex interactions between micro-organisms and hosts. ${ }^{25}$

Experimental laboratory-based studies allow biofilm experiments to be performed under controlled standardized conditions and allow for detailed research to be performed on the microbial response to independently modified nutrient conditions.

The aim of the study was to conduct a multiparametric investigation of S. mutans biofilm formation on clinically relevant human enamel surfaces after exposure to xylitol, sucrose and an endogenous glucose control. The biofilm viability was monitored by microbial cultivation. Furthermore, viability features including vitality, respiratory activity (5-cyano-2,3-ditolyl tetrazolium chloride, CTC), and the production of extracellular polysaccharides (EPS) were investigated separately in the inner, middle and outer layers of the biofilm. In addition to the biofilm structure, the metabolic sugar consumption (glucose and sucrose) of the S. mutans biofilm samples was determined. The expression of representative biofilm-associated genes was investigated. The genes were involved in (i) microbial adhesion (1a-sucrose-dependent adhesion: $g b p B, s a c B$ (ftf) vicR and wapA; 1bsucrose-independent adhesion: spaP) and biofilm formation (atlA, sacB (ftf), SMU.609, vicR and wapA); (ii) extracellular polysaccharide synthesis ( $g t f A, g t f B, g t f C, g t f D, s a c B$, $f t f$ and vicR); (iii) carbohydrate uptake (mipB, SMU.104, SMU.105 and sorA); and (iv) acid tolerance (comD and SMU.1037c).

\section{MATERIALS AND METHODS}

S. mutans cells were cultivated for $24 \mathrm{~h}$ in three different media: Schaedler medium (as a control; $0.58 \%$ endogenous glucose) (C), Schaedler medium $+5 \%$ sucrose (S) and Schaedler medium $+1 \%$ xylitol (X). Microbiological testing was performed for all experimental biofilms. Vitality, respiratory activity and EPS production in the inner, middle and outer biofilm layers were determined by confocal laser scanning microscopy (CLSM). The gene expression of representative genes was analysed using real-time quantitative polymerase chain reaction (RT-qPCR). The glucose concentrations (in C, S and X) and the sucrose concentrations (in S) were also quantified in sterile media and in the biofilms at $24 \mathrm{~h}$.

\section{Adhesion substrata for biofilms}

The adhesion substrata were prepared according to a method described previously. ${ }^{26}$ Intact human third molars were embedded in a methacrylate-based mounting material, sectioned longitudinally, fixed mechanically to glass and polished with a precision grinding machine. The surfaces were adapted to a surface roughness of $(0.19 \pm 0.03) \mu \mathrm{m}$, which corresponds to the magnitude of natural enamel-enamel contact $\operatorname{areas}^{27}(0-1 \mu \mathrm{m})$, and measured using a Perthometer (S6P; Mahr, Göttingen, Germany).

\section{Microbiology (culture, direct counts, $\mathrm{CFU}$, biofilm formation)}

A 50- $\mu \mathrm{L}$ aliquot of $S$. mutans cells (ATCC 25175) was inoculated in $5 \mathrm{~mL}$ of Schaedler broth (Becton Dickinson, Heidelberg, Germany) and grown for $8 \mathrm{~h}$ at $37^{\circ} \mathrm{C}$. A $1-\mathrm{mL}$ aliquot of the culture was then transferred to fresh Schaedler broth $(5 \mathrm{~mL})$ and grown for $17 \mathrm{~h}$ at $37{ }^{\circ} \mathrm{C}$. For biofilm formation, the $17 \mathrm{~h}$ overnight cultures were pooled, sonicated ( 20 pulses, $30 \mathrm{~W})$ and harvested by centrifugation. The seeded density corresponded to a concentration of approximately $10^{8} \mathrm{CFU} \cdot \mathrm{mL}^{-1}$. The pooled suspension was harvested and resuspended in three sugar-containing growth media: Schaedler broth with glucose (control, C), Schaedler broth supplemented with sucrose (S) and Schaedler broth supplemented with xylitol (X). Prior to use, the human enamel slides were conditioned in sterile Schaedler broth
( $1 \mathrm{~mL}$ for each slide) for $2 \mathrm{~min}$. Biofilms of $S$. mutans cells were cultured in microtiter plates under the three different dietary conditions by covering the preconditioned enamel slides with a $500 \mu \mathrm{L}$ bacterial suspension for $24 \mathrm{~h}$ at $37^{\circ} \mathrm{C}$.

Testing of both the planktonic bacterial and detached biofilm bacterial samples included the determination of the total bacterial cell count (BC) by dark field microscopy and of the bacterial growth in colony-forming units (CFU). Bacterial samples $(20 \mu \mathrm{L})$ were inoculated by spiral plating onto Schaedler agar plates in three dilution steps $\left(10^{-4}\right.$, $10^{-5}, 10^{-6}$ ) and incubated anaerobically for $48 \mathrm{~h}$ at $37^{\circ} \mathrm{C}$. The $\mathrm{pH}$ of the suspensions was measured before and after biofilm formation.

\section{In situ analysis of biofilms (vitality, CTC, EPS)}

CLSM analysis. S. mutans biofilms were analysed using a CLSM TCS SP5 (Leica Microsystems, Mannheim, Germany) equipped with an argon laser $(488 \mathrm{~nm})$, diode pumped solid state (DPSS) laser $(561 \mathrm{~nm})$ and HeNe laser $(633 \mathrm{~nm})$. The confocal images were obtained using a $\times 63$ water immersion objective. Serial optical sections were recorded at $1 \mu \mathrm{m}$ intervals in the $z$ direction throughout the biofilm from bottom to top. Line averaging $(\times 3)$ was used to improve the signal-to-noise ratio. The maximum biofilm thickness was measured, which corresponded to the number of slices per micrometre. The image frame was $512 \times 512$ pixels in size. For quantitative analysis of the vitality, respiratory activity and EPS production, each individual layer of the CLSM stacks was analysed. Data assessment and image processing were conducted using Axiovision 4.7.2.0 software (Carl Zeiss, Göttingen, Germany) complemented with a specially adapted macro software module. Data evaluation was performed using two different methods:

1. The mean fluorescence values were identified according to each confocal $z$-stack by averaging the fluorescence signals of single optical sections.

2. The biofilm data spatially resolved in the $z$ direction were evaluated.

The normalisation of the data from the inner, middle and outer biofilm compartments allowed for a comparison of biofilm stacks with different heights or structures.

Microbial vitality. The staining of microbial samples with the combination of two nucleic acid stains-Syto 9 (S9) and propidium iodide (PI) - from the Live/Dead BacLight Bacterial Viability Kit (Invitrogen, Darmstadt, Germany) indicated the membrane integrity status and allowed the differentiation between living (intact membranes, green fluorescence) and non-vital/dead (compromised membranes, red fluorescence) cells. $^{28}$ The fluorescent staining of the biofilm samples was conducted using S9 (argon laser, excitation: $488 \mathrm{~nm}$ ) and propidium iodide (DPSS laser, excitation: $561 \mathrm{~nm}$ ). The staining solution was prepared by mixing $5.0 \mu \mathrm{mol} \cdot \mathrm{L}^{-1} \mathrm{~S} 9$ and $30.0 \mu \mathrm{mol} \cdot \mathrm{L}^{-1} \mathrm{PI}$ in ultrapure water. At $24 \mathrm{~h}$, the biofilms were slightly dipped into sterile water to remove unbound cells and were subsequently covered with staining solution for $15 \mathrm{~min}$ in darkness. Optical sections were obtained sequentially to avoid bleed-through artefacts caused by overlapping excitation/emission spectra of the fluorophores. The percentage of vital streptococci was calculated as follows:

$$
\frac{[\operatorname{vital}(\mathrm{S} 9, \text { green }) \text { bacteria }]}{[\operatorname{vital}(\mathrm{S} 9, \text { green })+\operatorname{dead}(\mathrm{PI}, \text { red }) \text { bacteria }]} \times 100 \%
$$

Respiratory activity (CTC). Healthy respiring biofilm bacteria were identified by applying CTC (Invitrogen, Darmstadt, Germany) and by 
microbial counterstaining with S9. The redox dye CTC, a non-fluorescing stain, is absorbed and reduced into an insoluble, red fluorescent formazan product by metabolically active cells respiring via the electron transport chain. The biofilm samples were stained with $4.8 \mathrm{mmol} \cdot \mathrm{L}^{-1}$ CTC in PBS/Schaedler $(1: 1)$ and $5.0 \mu \mathrm{mol} \cdot \mathrm{L}^{-1} \mathrm{~S} 9$ for $1 \mathrm{~h}$ at $37^{\circ} \mathrm{C}$. The excitation of CTC and S9 was performed using a $561 \mathrm{~nm}$ DPSS laser and a $488 \mathrm{~nm}$ argon laser. The CLSM stacks were acquired sequentially. The percentage of the respiratory activity was calculated as:

$$
\frac{[\text { respiring }(\mathrm{CTC}, \text { red }) \text { bacteria }]}{[\text { total }(\mathrm{S} 9, \text { green })+\text { respiring }(\mathrm{CTC}, \text { red }) \text { bacteria }]} \times 100 \%
$$

EPS production (concanavalin A). For imaging the EPS production, S. mutans biofilms were stained with $0.96 \mu \mathrm{mol} \cdot \mathrm{L}^{-1}$ Alexa Fluor 633, labelled lectin concanavalin A (Con A), immersed in ultrapure water for one hour, and counterstained with $5.0 \mu \mathrm{mol} \cdot \mathrm{L}^{-1} \mathrm{~S} 9$ for $15 \mathrm{~min}$. This type of lectin represents a marker for glycoconjugates, which are major components of the EPS, and binds selectively to $\alpha$-glucopyranosyl molecules such as glucans. The fluorescent dyes were excited by the argon laser (488 nm, S9) and the HeNe laser (633 nm, EPS-Alexa Fluor 633). The percentage of EPS was calculated as follows:

[EPS-related fluorescence(Con A, red)] $\overline{[\operatorname{bacterial}(\mathrm{S} 9, \text { green })+\text { EPS-related fluorescence }(\text { Con A, red })]} \times 100 \%$

\section{Sugar consumption}

Biochemical glucose/sucrose assay. The glucose and sucrose analyses were based on the oxidation reactions of glucose and of the glucose molecules released from sucrose, which generated a dye (Resorufin) that could be detected in a colorimetric assay at $570 \mathrm{~nm}$ (Glucose Assay Kit, Glucose and Sucrose Assay Kit; BioVison, Mountain View, CA, USA). Three calibration curves were generated for each glucose and sucrose. Representatively, three glucose analyses were conducted using sterile $\mathrm{C}$ and $\mathrm{X}$ media, and three sucrose analyses were conducted using sterile S medium. The glucose/sucrose values from the sterile media served as a $100 \%$ control (time: $0 \mathrm{~h}$ ). After biofilm formation $(24 \mathrm{~h})$, the glucose and sucrose concentrations of three representative samples of $\mathrm{C}, \mathrm{S}$ and $\mathrm{X}$ were used to determine the metabolic consumption by streptococci (Table 1). All samples were tested in duplicate.

Glucose assay. The glucose assay was applied to determine the amount of free glucose in the samples in the $\mathrm{C}$ and $\mathrm{X}$ media. A glucose solution of $55.5 \mathrm{mmol} \cdot \mathrm{L}^{-1}$ in pure water was used as a standard for the glucose measurements. The calibration curves (absorbance vs. concentration) were prepared with concentrations of $0,0.8,1.7,2.5$, $3.3,4.2,5.0,5.8,6.7,7.5$ and $8.3 \mathrm{nmol}$ in $50 \mu \mathrm{L}$ of sample volume. After the colorimetric reaction, the optical density (OD) was measured every 5 min during a period of $30 \mathrm{~min}$ at $570 \mathrm{~nm}$ using a microplate reader (Synergy HT, BioTek Instruments $\mathrm{GmbH}$, Bad Friedrichshall, Germany) according to the manufacturer's instructions. Samples of sterile media were prediluted in pure water (C and X: $1: 50, S: 1: 300)$. The biofilm samples of $\mathrm{C}$ and $\mathrm{X}$ were measured undiluted, while the $S$. mutans supernatants from $S$ were diluted by a factor of $1: 100$. Each experimental run was accompanied by internal glucose standards at concentrations of $0,1.7,4.2$ and $6.7 \mathrm{nmol}$ in $50 \mu \mathrm{L}$. Blank values were obtained from the zero-glucose sample.

Sucrose assay. The sucrose assay was applied only to the samples from medium S, and it involved two main steps: (i) the detection of unspecific glucose in the sample $\left(\right.$ Glucl $\left._{\text {unspecific }}\right)$; and (ii) sucrose hydrolysis (by the addition of invertase) to fructose and sucrose-released glucose (Gluc2 $\left.2_{\text {sucrose }}\right)$. The total glucose concentration $\left(\mathrm{Gluc}_{\text {total }}=\mathrm{Glucl}_{\text {unspecific }}+\right.$ Gluc2 $\left.2_{\text {sucrose }}\right)$ was calculated according to the glucose and sucrose assay instructions. The sucrose-specific glucose was calculated as Gluc $2_{\text {sucrose }}=$ Gluc3 $3_{\text {total }}-$ Glucl $_{\text {unspecific }}$ In the colorimetric assay used to generate the calibration curves, the sucrose standard $\left(100 \mathrm{mmol} \cdot \mathrm{L}^{-1}\right.$ in pure water) was diluted to $0,1.0,2.0,3.0,4.0,5.0,6.0,7.0,8.0,9.0,10.0$ and $11.0 \mu \mathrm{mol}$ in $50 \mu \mathrm{L}$ sample volume. Both the first (unspecific glucose) and the second (sucrose-specific glucose) plate incubations lasted $30 \mathrm{~min}$ at $37{ }^{\circ} \mathrm{C}$, and for $30 \mathrm{~min}$ afterwards, the optical density was measured kinetically every $5 \mathrm{~min}$ at $37^{\circ} \mathrm{C}$. The processed data corresponded to the endpoint kinetic measurement obtained at $30 \mathrm{~min}$. For depicting the calibration curve of glucose/sucrose, a regression line was calculated to best fit the data. All optical density values of the biofilm supernatants were blank-corrected.

\section{Gene expression}

RNA isolation. After detachment from the enamel slides, the 24-h S. mutans biofilms were incubated in $1400 \mu \mathrm{L}$ of RNAprotect Bacteria Reagent (Qiagen $\mathrm{GmbH}$, Hilden, Germany) for $5 \mathrm{~min}$ at room temperature. After harvesting the bacteria by centrifuging cultures at $4800 \mathrm{~g}$ for $10 \mathrm{~min}$, the bacterial pellet was suspended in $1500 \mu \mathrm{L}$ of ice-cold phosphate buffer solution (PBS). The suspension was sonicated for $30 \mathrm{~s}$ and centrifuged at $5500 \mathrm{~g}$ for $10 \mathrm{~min}$ at $4{ }^{\circ} \mathrm{C}$. The washing step was repeated twice. The pellet was suspended in $700 \mu \mathrm{L}$ of RNA lysis buffer for lysing cells and tissues (RLT buffer; Qiagen $\mathrm{GmbH}$, Hilden, Germany), mixed and transferred to a lysing matrix tube (MP Biomedicals Europe, Illkirch, France). The bacterial cells were homogenized and lysed for $40 \mathrm{~s}$ at $6.0 \mathrm{~m} \cdot \mathrm{s}^{-1}$ using the FastPrep instrument (MP Biomedicals Europe, Illkirch, France) and subsequently cooled on ice for $1 \mathrm{~min}$. The homogenising step was repeated twice. After centrifugation at $12000 \mathrm{~g}$ for $5 \mathrm{~min}$ at room temperature, the supernatant was transferred into a fresh tube, and the bacterial RNA was isolated as described in the RNeasy Micro Kit instructions (Qiagen $\mathrm{GmbH}$, Hilden, Germany). The integrity of the total RNA obtained was measured using denaturing agarose gel electrophoresis. The quantity and purity of the RNA samples were assessed by ultraviolet (UV) spectroscopy.

$\boldsymbol{R} T$ - $\boldsymbol{q} P \boldsymbol{P C R}$. One microgram of total RNA was reverse-transcribed using random pentadecamer primers and the Omniscript RT Kit (Qiagen

Table 1 Concentrations of glucose, sucrose, and metabolic consumption by $S$. mutans biofilms in control, sucrose and xylitol media presented as reference values of sterile media and values of $S$. mutans supernatants after $24 \mathrm{~h}$ (mean \pm standard deviation)

\begin{tabular}{|c|c|c|c|c|c|c|c|c|}
\hline $\begin{array}{l}\text { Glucose and } \\
\text { sucrose }\end{array}$ & $\begin{array}{l}\text { Sterile medium } \\
\text { glucose } \\
\left.\text { conc./(g. } \mathrm{L}^{-1}\right)\end{array}$ & $\begin{array}{l}\text { Sm } 24 \mathrm{~h} \\
\text { glucose } \\
\left.\text { conc./(g. } \mathrm{L}^{-1}\right)\end{array}$ & $\begin{array}{c}S m 24 \mathrm{~h} \\
\text { residual glucose } \\
\text { conc. } / \%\end{array}$ & $\begin{array}{c}\text { Glucose } \\
\text { consumption/\% }\end{array}$ & $\begin{array}{l}\text { Sterile medium } \\
\text { sucrose } \\
\left.\text { conc./(g. } \mathrm{L}^{-1}\right)\end{array}$ & $\begin{array}{l}\text { Sm } 24 \mathrm{~h} \\
\text { sucrose } \\
\text { conc. } /\left(\mathrm{g} \cdot \mathrm{L}^{-1}\right)\end{array}$ & $\begin{array}{c}S m 24 \mathrm{~h} \\
\text { residual sucrose } \\
\text { conc. } / \%\end{array}$ & $\begin{array}{c}\text { Sucrose } \\
\text { consumption/\% }\end{array}$ \\
\hline Control & $5.81 \pm 0.60$ & $0.01 \pm 0.00$ & $0.12 \pm 0.04$ & $99.88 \pm 0.04$ & & & & \\
\hline Sucrose & $6.48 \pm 0.71$ & $0.01 \pm 0.00$ & $0.12 \pm 0.04$ & $99.88 \pm 0.04$ & $38.29 \pm 3.26$ & $19.05 \pm 4.92$ & $49.75 \pm 12.84$ & $50.25 \pm 12.84$ \\
\hline Xylitol & $5.94 \pm 0.68$ & $0.14 \pm 0.12$ & $2.32 \pm 2.07$ & $97.68 \pm 2.07$ & & & & \\
\hline
\end{tabular}

Sm, Streptococcus mutans; conc., concentration. 
GmbH, Hilden, Germany). The qPCR was performed using an iCycler real-time PCR detection system with iQ SYBR Green Supermix (BioRad Laboratories $\mathrm{GmbH}$, Munich, Germany). The total reaction volume was $25 \mu \mathrm{L}$, the template volume was $5 \mu \mathrm{L}$, and the final primer concentration was $500 \mathrm{nmol} \cdot \mathrm{L}^{-1}$ for both the sense and antisense primers according to the manufacturer's instructions. The cycling conditions were as follows: $5 \mathrm{~min}$ of initial denaturation at $95{ }^{\circ} \mathrm{C}$, 40 cycles consisting of $15 \mathrm{~s}$ at $95{ }^{\circ} \mathrm{C}$ and $60 \mathrm{~s}$ at $60{ }^{\circ} \mathrm{C}$, and a final melting curve program. The primers published by Shemesh et al. ${ }^{18}$ were adapted and verified for the above-mentioned cycler and cycling conditions. Amplifications using total RNA that was not reverse transcribed were performed to check for genomic DNA contamination, and no-template controls were included. The comparative $\Delta \Delta C_{\mathrm{T}}$ method for qPCR data was applied as a standard procedure in the analysis of relative gene expression data. ${ }^{29}$ The $C_{\mathrm{T}}$ values obtained from the experimental RNA samples were normalized to the reference gene $16 S$ rRNA. The difference in the $\Delta C_{T}$ values $\left(\Delta \Delta C_{T}\right)$ between the samples of interest and the control samples was calculated. The fold-change in the expression of the gene of interest is then equal to $2^{-\Delta \Delta C_{\mathrm{T}}} .^{29}$

\section{Statistical analysis}

Ten series of independent experiments were conducted for each nutrient condition ( $n=30$ biofilm samples, $n=90$ CLSM samples, $n=30$ gene expression samples, $n=9$ samples for biochemical sugar analysis). The BC and CFU data were lg-transformed. The statistical analysis was performed using one-way analysis of variance followed by the
Tukey-Kramer test at a significance level of $\alpha=0.05$. The figures display the mean and mean-based $95 \%$ confidence intervals of analysis of variance. Statistical significance is denoted by asterisks.

\section{RESULTS}

Microbiology (culture, direct counts, CFU)

The BC. $\mathrm{mL}^{-1}$ and CFU $\cdot \mathrm{mL}^{-1}$ output values of the suspended $\mathrm{C}, \mathrm{S}$ and $\mathrm{X}$ cultures were in the same order of magnitude $(\mathrm{lg} \mathrm{BC}$ per $\mathrm{mL}$ in $\mathrm{C}$ : 8.41 \pm 0.10 , S: $8.37 \pm 0.11, \mathrm{X}: 8.41 \pm 0.07$; lg CFU per $\mathrm{mL}$ in C: $8.52 \pm 0.17$, $\mathrm{S}: 8.49 \pm 0.12, \mathrm{X}: 8.51 \pm 0.11)$. The $\mathrm{pH}$ values at the experimental start


biofilm formation, $\mathrm{pH}$ values were significantly different: The lowest value occurred in $\mathrm{S}(4.28 \pm 0.05)$, while the highest value occurred in $\mathrm{C}$ (4.54 \pm 0.10$)$. The $\mathrm{pH}$ of $\mathrm{X}$ was between that of $\mathrm{S}$ and $\mathrm{C}(4.38 \pm 0.05)$. The BC and CFU per $\mathrm{mm}^{2}$ of the biofilm samples did not show statistically significant differences between the three growth media $\left(\mathrm{BC} \cdot \mathrm{mm}^{-2}\right.$; C: $6.94 \pm 0.04, \mathrm{~S}: 6.93 \pm 0.04, \mathrm{X}: 6.83 \pm 0.004),\left(\mathrm{CFU} \cdot \mathrm{mm}^{-2}\right.$; C: $6.95 \pm 0.05$, S: $6.90 \pm 0.05, \mathrm{X}: 6.84 \pm 0.05)$.

\section{In situ biofilm analysis (vitality, CTC, EPS)}

The combined data analysis of all biofilm samples based on confocal laser scanning microscopy revealed that there was no difference in the thickness of the biofilm samples produced in the different media $(\mu \mathrm{m}$;

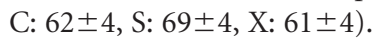

Specifically, $S$. mutans biofilms grown in the presence of sucrose were characterized by densely clustered microcolonies (Figure $1 \mathrm{~b}, 1 \mathrm{e}$ and $1 \mathrm{~h}$ ).
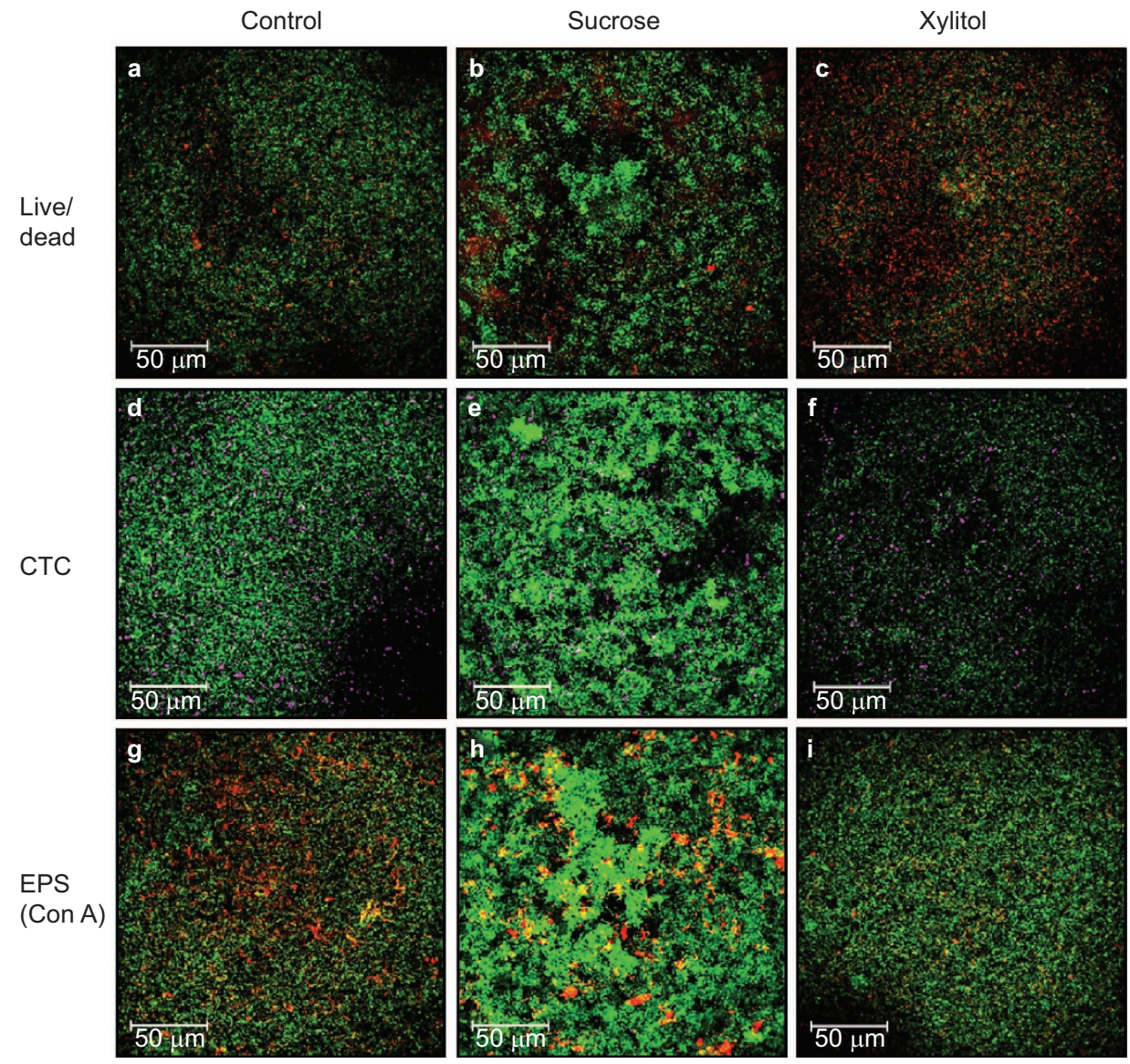

Figure 1 CLSM images (maximum projection) of 24-h S. mutans biofilms on enamel slides. (a-c) Vitality (live/dead) of biofilms grown in the control medium, sucrose medium, and xylitol medium; (d-f) respiratory activity (CTC) of biofilms grown in the control medium, sucrose medium and xylitol medium; (g-i) EPS production (Con A) in biofilms grown in the control medium, sucrose medium and xylitol medium. Con A, concanavalin A; CTC, 5-cyano-2,3-ditolyl tetrazolium chloride; EPS, extracellular polysaccharide. 


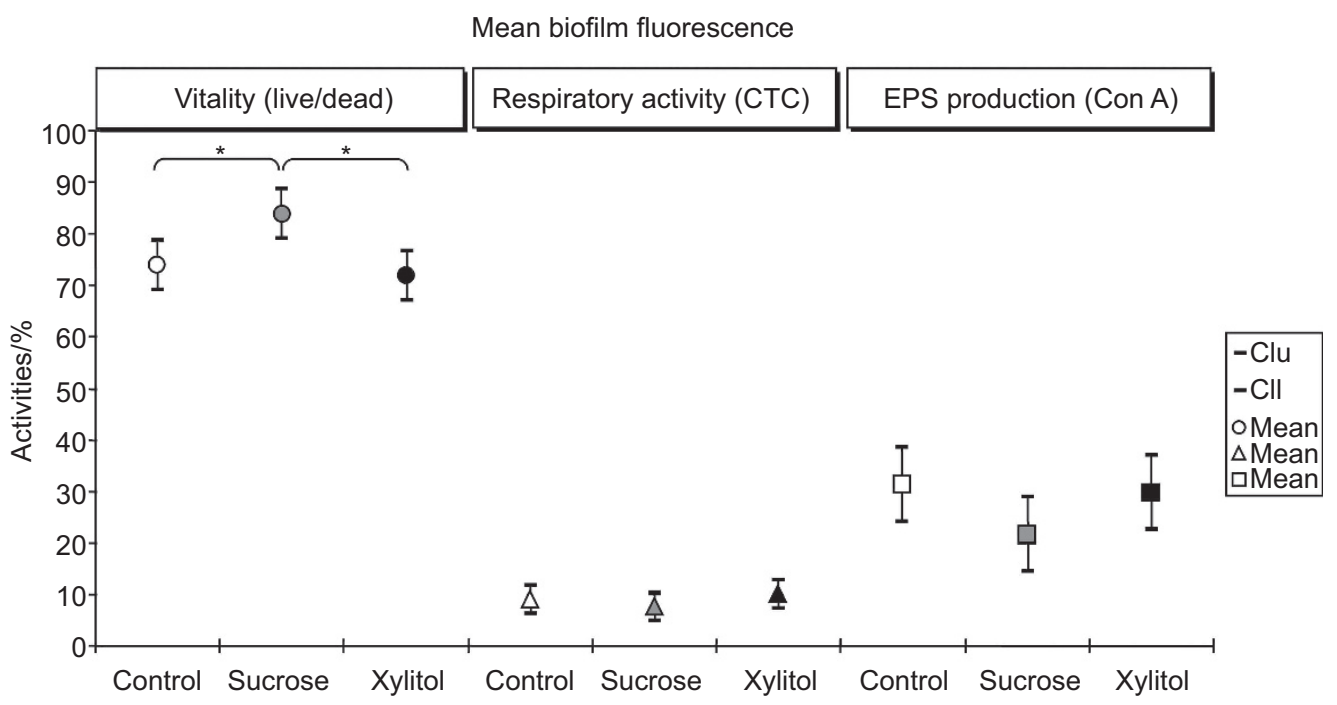

Figure 2 Mean values of biofilm fluorescence activities (vitality, CTC activity, EPS production) in S. mutans biofilm stacks after $24 \mathrm{~h}$ from the C (control), S (sucrose) and X (xylitol) conditions. Error bars indicate the $95 \%$ confidence interval. Statistical significance is denoted by asterisks $(n=10)$. Cll, lower limit of the $95 \%$ confidence intervals; Clu, upper limit of the 95\% confidence intervals; Con A, concanavalin A; CTC, 5-cyano-2,3-ditolyl tetrazolium chloride; EPS, extracellular polysaccharide.

Vitality. The exposure of S. mutans biofilms to xylitol and control levels of glucose yielded similar results for both the average vitality proportions (Figure 2) and the vitality proportions for the inner, middle and outer layers (Figure 3a). Concerning the average vitality percentages, the highest statistically significant values were found in the biofilms grown in the presence of sucrose (84\%) compared to those in the biofilms grown in the control medium $(74 \%)$ or the xylitol-supplemented medium (72\%), as illustrated in Figure 2. In regard to the spatial resolution, the local vitality after sucrose exposure in the inner, middle and outer biofilm regions (inner: $80 \%$, middle: $85 \%$, outer: $85 \%$ ) was higher and remained in a similar range throughout the biofilm. This result was in contrast to the lower vitality of the inner biofilm layers and increasing vitality percentages in the middle and outer layers of the biofilm in the control media (inner: 66\%, middle: 79\%, outer: $75 \%$ ) and in the media containing xylitol (inner: 62\%, middle: $78 \%$, outer: $79 \%$ ) (Figure 3a).

CTC activity. The average CTC activity was similar across all layers of the biofilms grown in the control, sucrose and xylitol media (Figure 2). The spatially resolved CTC profile did not indicate a nutrient dependency. Increased activity values could only be observed after glucose exposure in the inner layers compared to the CTC activity in the corresponding middle or outer biofilm layers (Figure $3 \mathrm{~b}$ ).

EPS production. The average EPS production of S. mutans biofilms grown in the glucose control, sucrose and xylitol media did not show significant differences (Figure 2). The locally resolved and Con Adetected EPS production of biofilms grown in the presence of sucrose was lower only in the inner and middle biofilm layers compared with that observed in the glucose exposure condition (Figure 3c). The mean EPS production and the layer-specific EPS production (for the inner, middle, and outer layers) of biofilm samples were similar under conditions of xylitol and glucose exposure (Figure 2 and 3c). The EPS production was at the same level in the outer biofilm layers regardless of the carbohydrate present.

\section{Sugar consumption}

The enzymatic detection of glucose and sucrose showed an almost complete metabolic consumption of glucose $(>97 \%-100 \%)$ by $S$. mutans cells in the 24-h biofilms grown in glucose-, sucrose- and xylitol-supplemented media (Table 1). At this growth stage, the sucrose consumption in the S-supplemented medium was approximately $50 \%$.

\section{Gene expression}

The genes $g b p B, s a c B$ ( $f t f)$, vicR and wapA are associated with sucrosedependent adhesion and were significantly upregulated in response to sucrose and xylitol supplementation compared to growth without supplementation (i.e., in the glucose control medium) (Figure 4). No significant differences were found in these genes in either sucroseor xylitol-supplemented media. In contrast, spaP, which is associated with sucrose-independent adhesion, was expressed at a significantly higher level in the xylitol-supplemented medium compared to the sucrose-supplemented and glucose control media. Nevertheless, the gene expression in the sucrose-supplemented medium was significantly upregulated compared to that in the control medium.

With the exception of SMU.609, all other genes related to biofilm formation ( $a t l A, s a c B(f t f)$, vicR, wapA) were also significantly upregulated in the sucrose- and xylitol-supplemented media compared to the glucose control medium. The difference in gene expression between these two media was not significant. With SMU.609, there was no difference between the xylitol-supplemented medium and the glucose control medium; however, in the sucrose-supplemented medium, the gene expression was significantly downregulated.

The genes $g t f D, s a c B(f t f)$ and $v i c R$ are all members of a group of genes related to EPS synthesis, and all were similarly upregulated to most of the biofilm group genes. Significant upregulation of these genes was thus observed in the sucrose- and xylitol-supplemented medium, but no difference was observed between these two groups compared to the glucose base medium. gtfC was only upregulated in the xylitol-supplemented medium. $g t f B$ was also upregulated in the xylitol-supplemented media, but in contrast to $g t f C$, its expression in the sucrose-supplemented medium was also upregulated. Nevertheless, 
Vitality (live/dead)

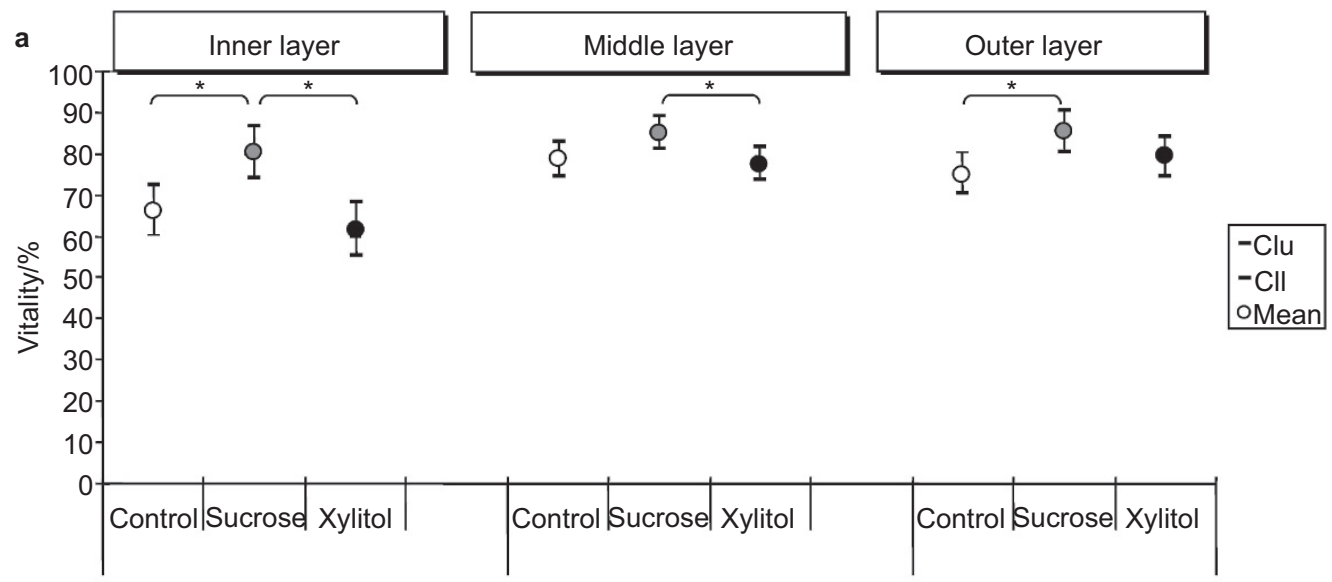

Respiratory activity (CTC)

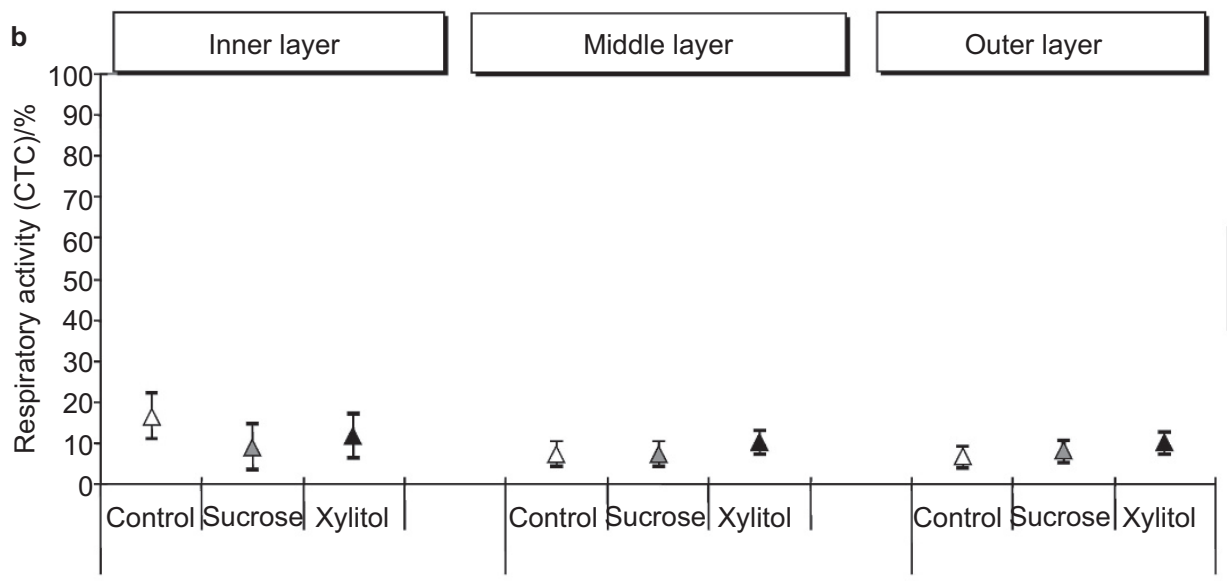

EPS production (Con A)

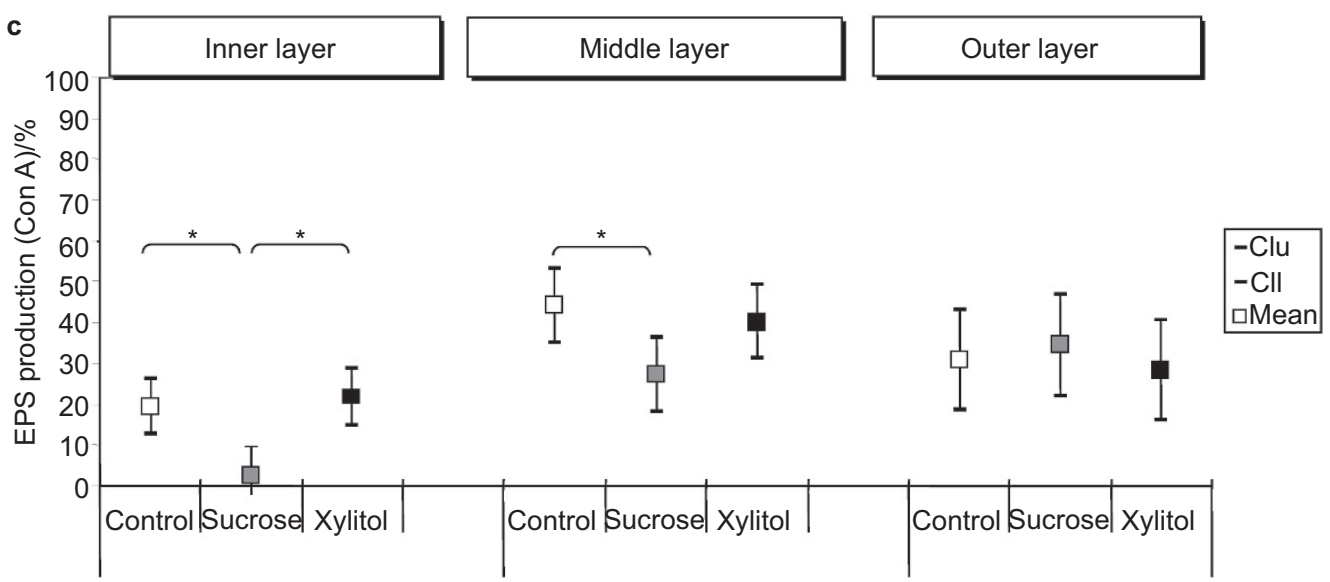

Figure 3 Biofilm fluorescence of the inner, middle, and outer layers in S. mutans biofilms after $24 \mathrm{~h}$ of growth in the C (control), S (sucrose) and X (xylitol) media. (a) Vitality (live/dead); (b) respiratory activity (CTC); (c) EPS production (Con A). Error bars depict the 95\% confidence interval. Statistical significance is denoted by asterisks $(n=10)$. Cll, lower limit of the 95\% confidence intervals; Clu, upper limit of the 95\% confidence intervals; Con A, concanavalin A; CTC, 5-cyano-2,3-ditolyl tetrazolium chloride; EPS, extracellular polysaccharide.

the upregulation of $g t f B$ in the xylitol-supplemented medium was twice that observed in the sucrose-supplemented medium; therefore, $g t f B$ was at a significantly higher level. gtf $A$ is the only gene in this group that was found to be downregulated in both the xylitol- and the sucrose-supplemented medium. Nevertheless, the downregulation in the sucrose-supplemented medium was twice that observed in the xylitol-supplemented medium and was therefore at a significantly lower level. The genes involved in carbohydrate uptake (mipB, SMU.104, SMU.105 and sorA) were predominantly upregulated in both types of supplemented media. An exception to this rule was the expression of mipB, which was significantly 

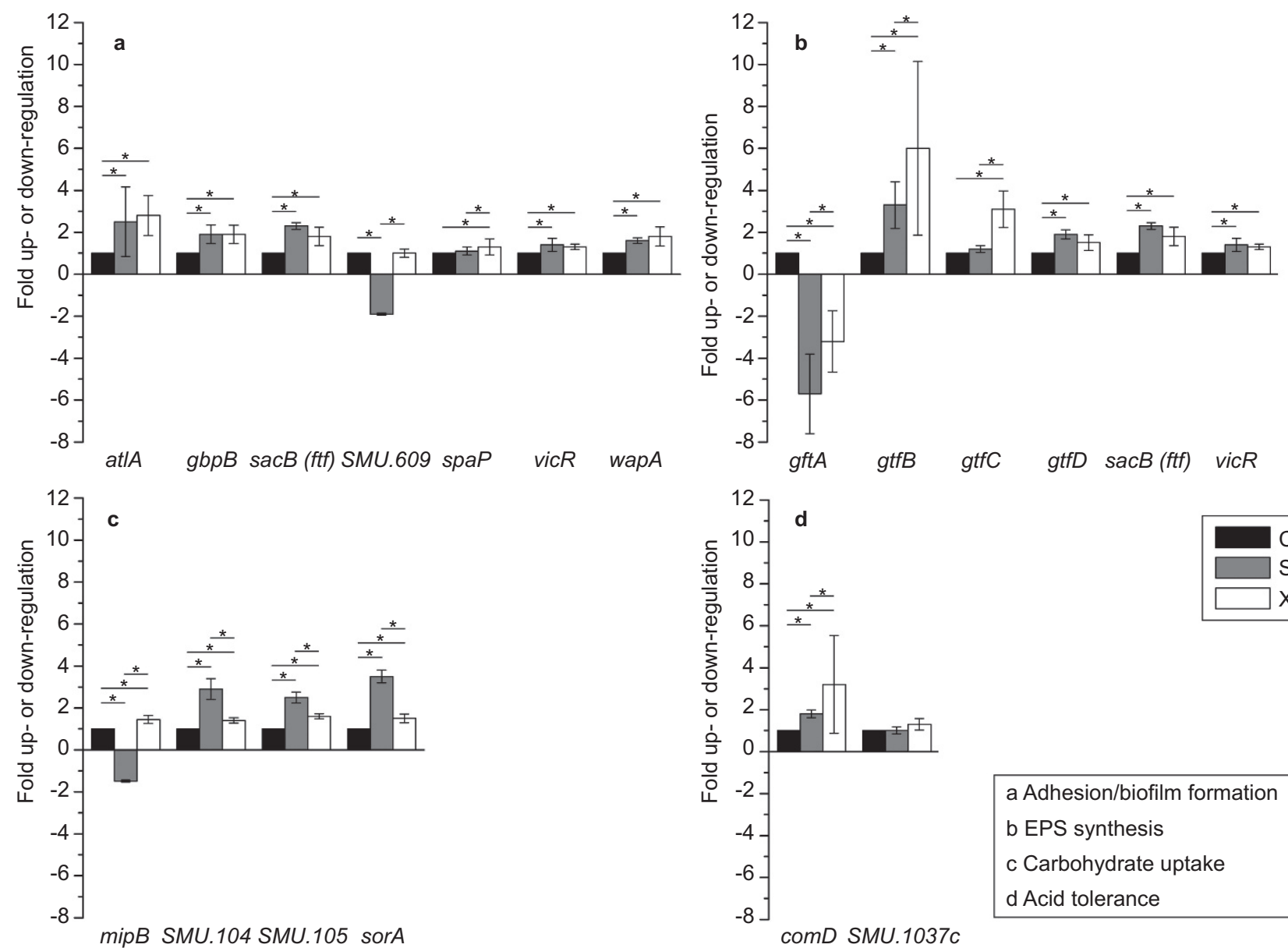

\section{a Adhesion/biofilm formation \\ b EPS synthesis \\ c Carbohydrate uptake \\ d Acid tolerance}

Figure 4 Expression of genes involved in four metabolic pathways in 24-h S. mutans biofilms. (a) Adhesion/biofilm formation; (b) EPS synthesis; (c) carbohydrate uptake; (d) acid tolerance. Fold upregulation or downregulation is depicted in relation to the unsupplemented base medium (control) containing $0.58 \%$ glucose. Error bars represent the $95 \%$ confidence interval. Statistical significance is denoted by asterisks $(n=10)$. EPS, extracellular polysaccharide.

downregulated in the sucrose-supplemented medium but upregulated in the xylitol-supplemented medium to the same level as the other genes in this group. The gene expression of the other genes of the group was upregulated significantly higher in the sucrose-supplemented medium than in the xylitol-supplemented medium. Nevertheless, the gene expression of the xylitol-supplemented medium was also significantly upregulated in comparison to the glucose control medium.

Finally, two members of two-component systems associated with acid tolerance were assessed. SMU.1037c was not upregulated in either type of supplemented media, whereas comD was upregulated in both types of supplemented media relative to the glucose control medium. Nevertheless, the gene expression of comD in the xylitol-supplemented medium was significantly higher than that in the sucrose-supplemented medium.

\section{DISCUSSION}

Although many clinical studies have assessed the effects of nutrient sweeteners on S. mutans, the therapeutic anti-caries efficacy of xylitol on streptococci has not been unequivocally clarified. In the present study, for the first time, a multifaceted approach was developed that combines analytical methods, such as scaling the CLSM stacks in the inner, middle and outer biofilm compartments to determine spatial profiles of microbial vitality, respiratory activity and EPS synthesis, with gene expression analysis and quantification of glucose/sucrose consumption by $S$. mutans biofilms under conditions of xylitol, sucrose and glucose exposure. The experimental biofilm model used in this study allowed the simulation of oral cariogenic conditions for $S$. mutans biofilm formation under standardized environmental conditions. The 5\% sucrose concentration used in the present study correlates with the sucrose threshold concentration for cariogenic biofilm formation that causes acid attack on and demineralisation of enamel. ${ }^{30-31}$ Aires et al. ${ }^{30}$ showed that $5 \%$ sucrose has the same cariogenic potential as $10 \%$ and $20 \%$ sucrose. With respect to xylitol, different concentrations were used in in vitro studies, ranging from $5 \%{ }^{32}$ to $1 \% .^{33-34}$ The concentration of $1 \%$ xylitol used in this study was based on several prior clinical studies, which analysed a final xylitol concentration of $1 \%$ in the saliva for $10 \mathrm{~min}$ after the use of xylitolcontaining products such as chewing gum or toothpaste. ${ }^{35-36}$ Schaedler broth, the growth medium used for S. mutans, contained endogenous glucose as an essential carbohydrate source and served as a control medium in this study. It also served as the basic medium for supplementation with sucrose and xylitol to produce media S and X, respectively. Therefore, endogenous glucose is also present in the sucrose- and xylitol-supplemented media.

\section{Xylitol effects}

The findings of the present study indicate that under the experimental conditions, xylitol (in the presence of glucose) did not inhibit the growth of $S$. mutans biofilm cells. The microbiological data indicated 
similar metabolic activities after xylitol exposure compared to the glucose control and sucrose conditions. The vitality of biofilms grown in xylitol and glucose was statistically decreased in the inner layer compared with the middle and outer layers. However, it is possible that the availability of carbohydrate substrates decreased from outer to inner biofilm layers due to a declining nutrient concentration.

The quantitative carbohydrate analysis of glucose in S. mutans biofilms supplemented with xylitol showed a high microbial metabolism of glucose that was similar to that observed in the reference medium. These findings, in addition to the metabolic activity profiles, suggest that the $S$. mutans biofilm cells are at a similar metabolic stage as those in the reference medium containing glucose after $24 \mathrm{~h}$ of biofilm formation.

Similar to sucrose, xylitol induced the expression of several carbohydrate-associated genes involved in adhesion, biofilm formation and EPS synthesis in S. mutans biofilms. However, the extent to which these normalized gene expression levels changed differed: gtfB was upregulated sixfold in response to xylitol (and threefold in response to sucrose), whereas comD was upregulated threefold in response to xylitol (and twofold in response to sucrose). The genes gtfC (sucrosedependent adhesion $^{37}$ ) and spaP (sucrose-independent adhesion ${ }^{38}$ ) were upregulated only after xylitol exposure. These results demonstrate that $g t f C$ was only upregulated in the presence of xylitol and are consistent with the work of Shemesh et al. ${ }^{18}$ Regarding acid tolerance, xylitol exposure induced significant upregulation of $\operatorname{comD}$ compared to sucrose exposure.

Although many research efforts have been devoted to clarifying the inhibitory effect of xylitol, the underlying plaque-reducing mechanisms have not yet been fully elucidated. The cellular uptake of xylitol by means of the flexible phosphotransferase system is an important step in the energy-consuming yet futile cycle, which is believed to be responsible for its inhibitory effect. ${ }^{39-40}$ Xylitol 5-phosphate may compete with phosphofructokinase and may thus inhibit glycolysis. Depending on the available energy, xylitol may be phosphorylated intracellularly. Because of its cellular toxicity, xylitol 5-phosphate must be dephosphorylated and expelled as xylitol, requiring a major energy input. Furthermore, xylitol seems to cause a disruption of protein synthesis. ${ }^{8}$ As a metabolic consequence, several studies have shown that $S$. mutans cells reduced their growth and thereby decreased the production of both acid and extracellular polysaccharides. ${ }^{20,41}$ The effects and clinical efficiency of xylitol on oral biofilms are still controversial. Several studies have shown that the regular and long-term application of xylitol has a caries-preventive effect. ${ }^{20,41}$ Other studies could not confirm its anti-caries effects. ${ }^{42}$ Only a few studies are available concerning the effects of xylitol on S. mutans biofilms in vitro, ${ }^{32-33}$ and these have shown contradictory results as well. Badet et al. ${ }^{33}$ demonstrated the inhibitory influence of xylitol on model-based multispecies biofilms, whereas a study by Giertsen ${ }^{23}$ did not support the inhibitory effects of S. mutans cells, although this work also used a multispecies biofilm model with repeated xylitol exposure. The results of different studies are difficult to compare because the experimental conditions, bacterial isolates and procedures used can differ substantially.

In the present study, the different expression patterns of genes involved in carbohydrate metabolism observed in xylitol-associated biofilms indicated that in the late stationary growth phase, the observed glucose depletion in the presence of xylitol may induce changes in gene regulatory processes. This was evidenced by the upregulation of $g t f B, g t f C$ and $\operatorname{comD}$, causing a metabolic shift. These results may indicate that bacterial cells exposed to xylitol attempt to compensate for the increasing metabolic imbalance and counteract the negative energy balance at the gene expression level.
The uptake of xylitol into $S$. mutans cells was shown to be mediated by a specific fructose phosphotransferase system. ${ }^{43}$ In the presence of fructose, xylitol did not show growth inhibition, likely because the fructose phosphotransferase system is dependent on fructose for its affinity. Recently, other streptococci, such as Streptococcus pneumoniae, were investigated to determine the effects of xylitol, glucose and fructose on biofilm formation. ${ }^{44}$ The results showed that only xylitol by itself inhibited biofilm formation, whereas simultaneous exposure to xylitol and glucose produced no inhibitory effects. It is possible that under the conditions of the present study, the $S$. mutans cells responded to xylitol-glucose exposure similarly. These findings would explain the similar metabolic patterns of $S$. mutans biofilms grown in the reference medium and the xylitol-glucose medium for $24 \mathrm{~h}$.

It is conceivable that the differing clinical effects of xylitol that were observed in several studies might be partially linked to the fact that under the experimental conditions of some studies, xylitol was the only sweetener available, whereas in other studies, carbohydrates were added. Furthermore, the presence of varying nutritional carbohydrates in the oral cavity is difficult to control. In the future, greater attention should be devoted to the interactions between microbes and the environment because the metabolic response of streptococci seems to differ depending on whether it is grown in a pure or a mixed carbohydrate milieu.

\section{Sucrose effects}

In the present study, the microbiological data (the $\mathrm{BC}$ and $\mathrm{CFU}$ ) of $S$. mutans biofilms grown for $24 \mathrm{~h}$ indicated that growth was similar in all investigated media. Concerning the microbial vitality, sucrose induced the highest vital cell proportions in all biofilm layers and on average (compared to the control and xylitol-containing media). The EPS production of the sucrose-exposed biofilms was similar to that of biofilms exposed to other nutrient media, with the exception of the observed lower values in the inner biofilm layers. The glucose consumption in the sucrose-supplemented medium seemed to follow a similar pattern as that in the glucose control or xylitol-containing media. The lower EPS levels in the sucrose-supplemented S. mutans biofilms in the inner layers after $24 \mathrm{~h}$ may be due to the preference of the microorganisms for quickly utilisable monosaccharides such as glucose. Only after the depletion of the preferred carbohydrate source does the microbe induce a time-consuming shift to sucrose metabolism.

The increased EPS production in the outer layers also seemed to be consistent with the microbial survival strategy needed for adhesion to surfaces, as mediated by EPS localized to the biofilm surface.

The production of EPS by microorganisms depends on the phases of growth, nutritional availability, and environmental conditions. ${ }^{45}$ The microbial survival strategy includes the expression of virulence factors, such as EPS, which are needed for bacterial adhesion under stress conditions; ${ }^{46}$ such factors are important for surviving the limited nutrient availability in the later stages of stationary growth. Decker et al. ${ }^{47}$ found increased EPS production in planktonic $S$. mutans cultures in the late stationary phase after more than $48 \mathrm{~h}$ of growth compared to the EPS production after only $24 \mathrm{~h}$.

The key role of sucrose has been well documented in the biofilm formation of $S$. mutans cells. ${ }^{5,48-50}$

The caries- and biofilm-related virulence factors $\mathrm{GtfB}, \mathrm{GtfC}$ and GtfD are the three glucosyltransferases produced by $S$. mutans and are predominantly involved in extracellular polysaccharide synthesis. ${ }^{51} \mathrm{GtfB}$ and $\mathrm{GtfC}$ produce water-insoluble EPS, which may serve as an anchor molecule for bacterial adhesion and colonisation. ${ }^{52}$ Under conditions of sucrose exposure, the expression of several $S$. 
mutans genes associated with adhesion, biofilm formation, EPS synthesis and carbohydrate uptake were altered (either upregulated or downregulated) compared to the control.

Gene expression analysis revealed that in contrast to $g t f C, g t f B, g t f D$ and $f t f$ were upregulated in the presence of sucrose compared to the reference medium. These results are in agreement with the study of Shemesh et al., ${ }^{18}$ which reported the corresponding upregulation of $g t f B$ and $f t f$ in the late exponential growth phase in the presence of sucrose. Compared to xylitol supplementation, sucrose supplementation induced further differences in the expression profiles of genes related to biofilm formation and carbohydrate uptake: (i) SMU.609, which is involved in biofilm formation, was downregulated; (ii) gtfA, which is involved in EPS synthesis, was even more strongly downregulated; (iii) mip $B$, which is involved in carbohydrate update, was downregulated; and (iv) SMU_104, SMU_105 and sorA, all of which are also involved in carbohydrate uptake, were upregulated.

Although the corresponding CLSM images in the present study give the impression of dense clusters of biofilms, the biofilm thickness under conditions of sucrose exposure was not confirmed to be significantly different from that of the biofilms grown in the presence of glucose and xylitol. The phenotypic structure of the $S$. mutans biofilms showed distinctive microcolonies only after sucrose supplementation, which is consistent with the findings of earlier studies. ${ }^{9,10,53}$ Thus, the regulation of the glucosyltransferase $\mathrm{GtfB}$ seems to play a crucial role. ${ }^{54}$

\section{In situ activity profiles}

The metabolic detection of microbial vitality, respiratory activity and EPS production at different biofilm depths (the inner layer, which is exposed to the adhesion substratum, the middle layer, which is located between the material surface and the nutrient solution, and the outer layer, which is exposed to the nutritional environment) offers new insights concerning the activities within the biofilm. Both the spatially resolved vitality and EPS pattern observed in biofilms grown under all nutrient conditions indicated a tendency toward lower proportions in the inner layers compared to the middle and outer layers. One reason could be that the availability of carbohydrate substrates decreased due to the declining concentration gradient from the outer to the inner biofilm layers. The average respiratory activity detected by CTC did not show nutrient-dependent differences. Instead, the local patterns revealed a higher CTC rate only in the inner layer of biofilms grown in the glucose control condition compared to the corresponding middle or outer biofilm layers. It is conceivable that under conditions of glucose starvation, unknown metabolic activities occur inside the $S$. mutans biofilms, which also induce lower vitalities as a result.

Only a few studies have previously investigated spatially resolved metabolic activity. Gu et al. ${ }^{55}$ showed that the vitality of the inner layers of clinical biofilms exposed to a negative control was decreased compared with that of the other layers. This is in agreement with the results of the present study, although the adhesion experiments of $\mathrm{Gu}$ et al. ${ }^{55}$ were conducted on glass surfaces. This phenomenon also seems to be valid for non-S. mutans microorganisms, such as plaque bacteria. A different vitality pattern was reported by Zaura-Arite et al., ${ }^{56}$ who found similar vitality values throughout the clinical biofilm.

Despite the large number of studies dealing with the microbial interactions of cariogenic carbohydrates, there are limited data available concerning the quantification of sugar concentrations in the microbial environment.

Regarding the contradictory xylitol effects described in various in vivo and in vitro studies, causally related factors may include the composition of nutrients, the presence of single sugar components (or combinations thereof), and the genetic and metabolic responses. Further studies using multiparametric analytic methods and longer S. mutans biofilm maturation periods $(>24 \mathrm{~h}$ ) with exposure to pure and composed carbohydrates could provide additional information about the underlying regulatory mechanisms and metabolic activities.

In conclusion, $S$. mutans biofilms grown in xylitol-supplemented medium with endogenous glucose for $24 \mathrm{~h}$ did not show a reduction in the bacterial viability or metabolic activities, as measured by culture growth, vitality, respiratory activity, EPS production and glucose consumption, compared to biofilms grown in glucose-containing control medium evaluated at the same growth stage. In contrast, the upregulation of glucosyltransferases suggests that the combined presence of xylitol and glucose was effective in influencing the gene expression of glucan- and biofilm-associated genes in a different way than either sucrose or pure glucose.

At this growth stage, this observation may suggest that the compensation efforts of $S$. mutans cells to manage the energy status on a cellular level can be disturbed by the effects of xylitol. In the sucrosesupplemented nutrient solution, the S. mutans biofilms showed increased vitality throughout all biofilm layers and primary metabolic consumption of glucose with subsequent sucrose utilisation. Furthermore, sucrose-induced changes in biofilm morphology were observed, such as the development of microcolonies.

\section{ACKNOWLEDGEMENTS}

This study was supported by the National Deutsche Gesellschaft für Zahnerhaltung-GABA Scientific Fund, Germany.

1 ten Cate JM. Biofilms, a new approach to the microbiology of dental plaque. Odontology 2006; 94(1): 1-9

2 Hojo K, Nagaoka S, Ohshima T et al. Bacterial interactions in dental biofilm development. J Dent Res 2009; 88(11): 982-990.

3 Stoodley P, Sauer K, Davies DG et al. Biofilms as complex differentiated communities. Annu Rev Microbiol 2002; 56: 187-209.

4 Forssten SD, Björklund M, Ouwehand AC. Streptococcus mutans, caries and simulation models. Nutrients 2010; 2(3): 290-298.

5 Paes Leme AF, Koo H, Bellato CM et al. The role of sucrose in cariogenic dental biofilm formation-new insight. J Dent Res 2006; 85(10): 878-887.

6 Narisawa N, Kawarai T, Suzuki $\mathrm{N}$ et al. Competence-dependent endogenous DNA rearrangement and uptake of extracellular DNA give a natural variant of Streptococcus mutans without biofilm formation. J Bacteriol 2011; 193(19): 5147-5154

7 Takahashi N, Nyvad B. Caries ecology revisited: microbial dynamics and the caries process. Caries Res 2008; 42(6): 409-418.

8 Trahan L, Néron S, Bareil M. Intracellular xylitol-phosphate hydrolysis and efflux of xylitol in Streptococcus sobrinus. Oral Microbiol Immunol 1991; 6(1): 41-50.

9 Xiao J, Klein MI, Falsetta ML et al. The exopolysaccharide matrix modulates the interaction between 3D architecture and virulence of a mixed-species oral biofilm. PLoS Pathog 2012; 8(4): e1002623.

10 Klein MI, DeBaz L, Agidi S et al. Dynamics of Streptococcus mutans transcriptome in response to starch and sucrose during biofilm development. PLoS One 2010; 5(10): e13478.

11 Rolerson E, Swick A, Newlon L et al. The SloR/Dlg metalloregulator modulates Streptococcus mutans virulence gene expression. J Bacteriol 2006; 188(14): 5033-5044.

12 Senadheera MD, Guggenheim B, Spatafora GA et al. A VicRK signal transduction system in Streptococcus mutans affects gtfBCD, gbpB, and ftf expression, biofilm formation, and genetic competence development. J Bacteriol 2005; 187(12): 40644076

13 Shemesh M, Tam A, Steinberg D. Expression of biofilm-associated genes of Streptococcus mutans in response to glucose and sucrose. J Med Microbiol 2007; 56 (Pt 11): 1528-1535.

14 Shibata $\mathrm{Y}$, Kawada M, Nakano $\mathrm{Y}$ et al. Identification and characterization of an autolysin-encoding gene of Streptococcus mutans. Infect Immun 2005; 73(6): 3512-3520.

15 Banu LD, Conrads G, Rehrauer $\mathrm{H}$ et al. The Streptococcus mutans serine/threonine kinase, PknB, regulates competence development, bacteriocin production, and cell wall metabolism. Infect Immun 2010; 78(5): 2209-2220. 
16 Shemesh M, Tam A, Kott-Gutkowski M et al. DNA-microarrays identification of Streptococcus mutans genes associated with biofilm thickness. BMC Microbiol 2008; 8: 236 .

17 Sztajer H, Lemme A, Vilchez R et al. Autoinducer-2-regulated genes in Streptococcus mutans UA159 and global metabolic effect of the luxS mutation. J Bacteriol 2008; 190(1): 401-415.

18 Shemesh M, Tam A, Feldman M et al. Differential expression profiles of Streptococcus mutans ftf, gtfand vicRgenes in the presence of dietary carbohydrates at early and late exponential growth phases. Carbohydr Res 2006; 341(12): 2090-2097.

19 Gong Y, Tian XL, Sutherland T et al. Global transcriptional analysis of acid-inducible genes in Streptococcus mutans: multiple two-component systems involved in acid adaptation. Microbiology 2009; 155(Pt 10): 3322-3332.

20 Mäkinen KK. Sugar alcohol sweeteners as alternatives to sugar with special consideration of xylitol. Med Princ Pract 2011; 20(4): 303-320.

21 Tanzer JM. Xylitol chewing gum and dental caries. Int Dent J 1995; 45(1 Suppl 1): 65-76.

22 Trahan L. Xylitol: a review of its action on mutans streptococci and dental plaque-its clinical significance. Int Dent J 1995; 45(1 Suppl 1): 77-92.

23 Giertsen E, Arthur RA, Guggenheim B. Effects of xylitol on survival of mutans streptococci in mixed-six-species in vitro biofilms modelling supragingival plaque. Caries Res 2011; 45(1): 31-39.

24 Scheie AA, Fejerskov O, Danielsen B. The effects of xylitol-containing chewing gums on dental plaque and acidogenic potential. J Dent Res 1998; 77(7): 1547-1552.

25 Anderson SA, Sissons CH, Coleman MJ et al. Application of carbon source utilization patterns to measure the metabolic similarity of complex dental plaque biofilm microcosms. Appl Environ Microbiol 2002; 68(11): 5779-5783.

26 Decker EM, Weiger R, von Ohle $\mathrm{C}$ et al. Susceptibility of planktonic versus attached Streptococcus sanguinis cells to chlorhexidine. Clin Oral Investig 2003; 7(2): 98-102.

27 Willems G, Lambrechts P, Braem M et al. The surface roughness of enamel-to-enamel contact areas compared with the intrinsic roughness of dental resin composites. J Dent Res 1991; 70(9): 1299-1305.

28 Decker EM. The ability of direct fluorescence-based, two-colour assays to detect different physiological states of oral streptococci. Lett Appl Microbiol 2001; 33(3): 188-192.

29 Livak KJ, Schmittgen TD. Analysis of relative gene expression data using real-time quantitative PCR and the $2^{-\Delta \Delta C_{\mathrm{T}}}$ method. Methods 2001; 25(4): 402-408.

30 Aires CP. Tabchoury CP, del bel Cury AA et al. Effect of sucrose concentration on dental biofilm formed in situ and on enamel demineralization. Caries Res 2006; 40(1): 28-32.

31 Zhu M, Takenaka S, Sato M et al. Influence of starvation and biofilm formation on acid resistance of Streptococcus mutans. Oral Microbiol Immunol 2001; 16(1): 24-27.

32 Marttinen AM, Ruas-Madiedo P, Hidalgo-Cantabrana C et al. Effects of xylitol on xylitol-sensitive versus xylitol-resistant Streptococcus mutans strains in a threespecies in vitro biofilm. Curr Microbiol 2012; 65(3): 237-243.

33 Badet C, Furiga A, Thébaud N. Effect of xylitol on an in vitro model of oral biofilm. Oral Health Prev Dent 2008; 6(4): 337-341.

34 Söderling EM, Ekman TC, Taipale TJ. Growth inhibition of Streptococcus mutans with low xylitol concentrations. Curr Microbiol 2008; 56(4): 382-385.

35 Lif Holgerson P, Stecksén-Blicks C, Sjöström I et al. Xylitol concentration in saliva and dental plaque after use of various xylitol-containing products. Caries Res 2006; 40(5): 393-397.

36 Tapiainen T, Renko M, Kontiokari T et al. Xylitol concentrations in the saliva of children after chewing xylitol gum or consuming a xylitol mixture. Eur J Clin Microbiol Infect Dis 2002; 21(1): 53-55.

37 Hanada N, Kuramitsu HK. Isolation and characterization of the Streptococcus mutans gtfC gene, coding for synthesis of both soluble and insoluble glucans. Infect Immun 1988; 56(8): 1999-2005.

38 Nakano K, Nomura R, Nemoto $\mathrm{H}$ et al. Protein antigen in serotype $\mathrm{k}$ Streptococcus mutans clinical isolates. J Dent Res 2008; 87(10): 964-968.
39 Trahan L, Bareil M, Gauthier L et al. Transport and phosphorylation of xylitol by a fructose phosphotransferase system in Streptococcus mutans. Caries Res 1985; 19(1): 53-63.

40 Assev S, Rölla G. Further studies on the growth inhibition of Streptococcus mutans OMZ 176 by xylitol. Acta Pathol Microbiol Immunol Scand B 1986; 94(2): 97-102.

41 Lee YE, Choi YH, Jeong SH et al. Morphological changes in Streptococcus mutans after chewing gum containing xylitol for twelve months. Curr Microbiol 2009; 58(4): 332-337.

42 van Loveren C. Sugar alcohols: what is the evidence for caries-preventive and cariestherapeutic effects? Caries Res 2004; 38(3): 286-293.

43 Tapiainen T, Kontiokari T, Sammalkivi L et al. Effect of xylitol on growth of Streptococcus pneumoniae in the presence of fructose and sorbitol. Antimicrob Agents Chemother 2001; 45(1): 166-169.

44 Kurola P, Tapiainen T, Sevander J et al. Effect of xylitol and other carbon sources on Streptococcus pneumoniae biofilm formation and gene expression in vitro. APMIS $2011 ; 119(2): 135-142$.

45 Pal A, Paul AK. Optimization of cultural conditions for production of extracellular polymeric substances (EPS) by serpentine rhizobacterium Cupriavidus pauculus KPS 201. J Polym 2013; 2013: 692374.

46 Sims IM, Frese SA, Walter J et al. Structure and functions of exopolysaccharide produced by gut commensal Lactobacillus reuteri 100-23. ISME J 2011; 5(7): $1115-1124$

47 Decker EM, Dietrich I, Klein C et al. Dynamic production of soluble extracellular polysaccharides by Streptococcus mutans. Int J Dent 2011; 2011: 435830.

48 Bowen WH. Do we need to be concerned about dental caries in the coming millennium? Crit Rev Oral Biol Med 2002; 13(2): 126-131.

49 Pecharki GD, Cury JA, Paes Leme AF et al. Effect of sucrose containing iron (II) on dental biofilm and enamel demineralization in situ. Caries Res 2005; 39(2): 123129

50 Kreth J, Zhu L, Merritt J et al. Role of sucrose in the fitness of Streptococcus mutans. Oral Microbiol Immunol 2008; 23(3): 213-219.

51 Yamashita Y, Bowen WH, Burne RA et al. Role of the Streptococcus mutans gtf genes in caries induction in the specific-pathogen-free rat model. Infect Immun 1993; 61(9): 3811-3817.

52 Tsumori H, Kuramitsu H. The role of the Streptococcus mutans glucosyltransferases in the sucrose-dependent attachment to smooth surfaces: essential role of the GtfC enzyme. Oral Microbiol Immunol 1997; 12(5): 274-280.

53 Renye JA Jr, Piggot PJ, Daneo-Moore L et al. Persistence of Streptococcus mutans in stationary-phase batch cultures and biofilms. App/ Environ Microbiol 2004; 70(10): 6181-6187.

$54 \mathrm{Koo} \mathrm{H}$, Xiao J, Klein MI et al. Exopolysaccharides produced by Streptococcus mutans glucosyltransferases modulate the establishment of microcolonies within multispecies biofilms. J Bacteriol 2010; 192(12): 3024-3032.

$55 \mathrm{Gu} \mathrm{H}$, Fan D, Gao J et al. Effect of $\mathrm{ZnCl}_{2}$ on plaque growth and biofilm vitality. Arch Oral Biol 2012; 57(4): 369-375.

56 Zaura-Arite E, van Marle J, ten Cate JM. Conofocal microscopy study of undisturbed and chlorhexidine-treated dental biofilm. J Dent Res 2001; 80(5): 1436-1440.

\section{(i) $\Theta$ This work is licensed under a Creative Commons Attribution-}

c. party material in this article are included in the article's Creative Commons license, unles indicated otherwise in the credit line; if the material is not included under the Creative Commons license, users will need to obtain permission from the license holder to reproduce the material. To view a copy of this license, visit http://creativecommons.org/licenses/ by-nc-nd/3.0/ 